\title{
DEA Models for the Efficiency Evaluation of System Composed of Parallel Subsystems
}

\author{
Jinnian Wang ${ }^{*}$, Yongjun Li \\ School of Management, University of Science and Technology of China, Hefei, China \\ E-mail: *wjinnian@mail.ustc.edu.cn \\ Received August 30, 2011; revised September 27, 2011; accepted October 9, 2011
}

\begin{abstract}
The perspective of internal structure of the decision making units (DMUs) was considered as the "black box" when employing data envelopment analysis (DEA) models. However, in the actual world there are always some DMUs that are composed of several sub-units or subsystems, each utilizes the same inputs to generate same outputs. Numerous instances can be listed, such as a firm with a few of plants. In this paper we present models that evaluated the efficiency of DMU which is comprised of same several parallel subsystems, the foremost contribution of our work is that we take the different importance of the subsystems into account in the model, which can be obviously distinguished to the existing DEA model. Secondly, since the alternative optimal multipliers may emerge in the model, the efficiency of each subsystem may be non-unique and we simultaneously develop models of efficiency decomposition for each subsystem. At last a case of technological innovation activities of each province in China is used as an example to state the models.
\end{abstract}

Keywords: Data Envelopment Analysis, Parallel Subsystems, Efficiency Decomposition, Technological Innovation

\section{Introduction}

A non-parametric mathematical programming, data envelopment analysis (DEA), has been widely used in performance evaluation and benchmark [1]. Since the appearance of first CCR model [2], a series of different DEA models, such as BCC [3], FG [4], ST [5] and so on, have been proposed in succession. The method has been proved to be an effective tool, due to its advantage that it does not premeditate the production function, the intermediate process of inputs convert to outputs was treat as a "black box" manipulation.

Nevertheless, in the real world, it may be irrational for the efficiency evaluation of whole system when ignores its internal structure, especially which was composed of several subsystems. Scholars have committed to discuss the internal structure of the DMUs, a number of papers has done, such as two-stage model [6-9] of linear structure; network DEA model [10-12] with complicated internal structure. Moreover, system which is made up of several parallel subsystems have also been researched in many literatures, a model to measure the efficiency of multi-plant firms was proposed by Färe and Primont [13]; Yang [14] developed the so-called YMK model in meas- uring efficiencies of the production system with $\mathrm{k}$ independent subsystems. In addition, Castelli [15] present hierarchical structures of the DMU under appraisal. More recently, Kao [16] put forward a parallel DEA model which considers the operation of individual components to calculate the efficiency of the whole system.

In this paper, we will investigate the system with several parallel subsystems and assume that the number of the subsystems is same, which can depict as Figure 1. Each subsystem uses the same inputs to produce the same outputs, and cross utilization does not exist, the total inputs and outputs of the overall system are constituted by the sum of inputs and outputs of each subsystem. In summary, they are non-connatural in efficiency evaluation of whole system. However, to differentiate the above papers that assumed subsystems are equally important to the overall system, this paper believes that the importance of each subsystem may be different to a system under evaluation. For example, for some given fix resource, if one subsystem was considered to be more important, then more resource may be allocated to it rather than others, here the importance degree can represent by the volume of the inputs. Therefore, the different relative importance of each subsystem should be taking 


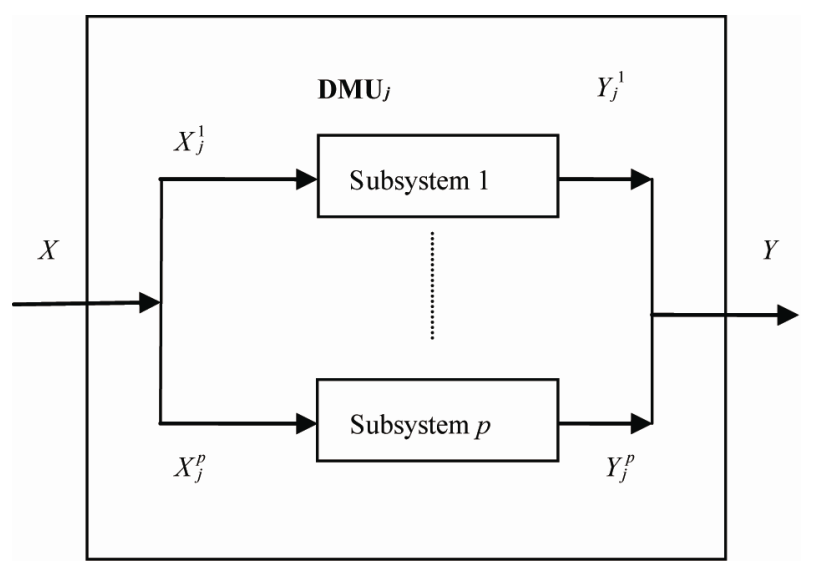

Figure 1. A parallel system composed of same subsystems.

into consideration in efficiency evaluation.

After measuring the efficiency score of the overall system, each subsystem was also investigated. The method of efficiency decomposition was applied to determine the efficiency of each subsystem in this paper, and rest is organized as follows: Section 2 develops DEA models for measuring the efficiencies of the overall system as well as each subsystem. In Section 3, an example of technological innovation activities of each region in China was used to illustrate the models introduced in Section 2. Finally, Section 4 gives our conclusions.

\section{DEA Models}

\subsection{Models for Measuring the Overall Parallel System}

To introduce the DEA model, suppose that there are a set of $n$ DMUs denoted by $\operatorname{DMU}_{j}(j=1, \cdots, n)$, each $\mathrm{DMU}_{j}(j=1, \cdots, n)$ composes of same p parallel components or subsystems and each used the same inputs to generate the same outputs. The subsystem $k$ of $\mathrm{DMU}_{j}(j=1, \cdots, n)$ contains $m$ inputs, denoted by $X_{i j}^{k}(i=1, \cdots, m)$ and $s$ outputs, denoted by $Y_{r i}^{k}(r=1, \cdots, s)$. The sums of $X_{i j}^{k}(k=1, \cdots, p)$ and $Y_{r j}^{k}(k=1, \cdots, p)$ are the whole inputs $X_{i j}(i=1, \cdots, m)$ and outputs $Y_{r j}(r=1, \cdots, s)$ of the jth parallel system, namely $\sum_{k=1}^{p} X_{i j}^{k}=X_{i j}(i=1, \cdots, m)$ and $\sum_{k=1}^{p} Y_{r j}^{k}=Y_{r j}(r=1, \cdots, s)$. Then, to any given $\mathrm{DMU}_{0}$, the efficiency of its subsystem $k(k=1, \cdots, p)$ can be calculated via a BCC model as following model (1).

$$
\max e_{0}^{k}=\frac{\sum_{r=1}^{s} u_{r}^{k} Y_{r 0}^{k}+\mu_{0}^{k}}{\sum_{i=1}^{m} w_{i}^{k} X_{i 0}^{k}} \forall k
$$

$$
\begin{array}{ll}
\text { s.t. } & \frac{\sum_{r=1}^{s} u_{r}^{k} Y_{r j}^{k}+\mu_{0}^{k}}{\sum_{i=1}^{m} w_{i}^{k} X_{i j}^{k}} \leq 1 \forall j \\
& w_{i}^{k}, u_{r}^{k} \geq 0, \forall r, i, \mu_{0}^{k}, \text { free }
\end{array}
$$

Here the optimal objective function value of model (2) was denoted as $e_{0}^{k}(k=1, \cdots, p)$, which is the efficiency value of each subsystem.

When we evaluate the performance of an overall parallel system, its inputs and outputs used in each subsystem are connatural, respectively, so this paper believes the weights attached to the same inputs and outputs in both subsystems are same, respectively. Therefore we have:

$$
w_{i}^{k}=w_{i}, \quad \forall i, k \text { and } u_{r}^{k}=u_{r}, \forall r, k
$$

The computation in (1) only considered each subsystem was equally. However, as we mentioned above, we believe that each subsystem may have different relative importance to a given DMU under evaluation. For example, if a company has two departments, sales department and production department, when the quantity supplied is more than quantity demanded, the manager of the company probable deemed that sales department is more relative important. Suppose that there are some given resources, such as human and fund, the manager may invest more resource in sales department rather than production department, it is obviously thought that the core activity of the company focus on marketing for the time being, if the two departments are treated as equal, the performance of the company was more impossible to become efficient. Therefore, the different relative importance of the two departments should be considered in the model. If we denote the relative importance of subsystem $k$ as $\lambda_{k}(k=1, \cdots, p)$, then $\lambda_{k}(k=1, \cdots, p)$ can be set as follows:

$$
\lambda_{\mathrm{k}}=\frac{\sum_{i=1}^{m} w_{i} X_{i 0}^{k}}{\sum_{i=1}^{m} w_{i} X_{i 0}}, \forall k
$$

The numerator and denominator in (3) denote the weighted gains of $\mathrm{DMU}_{0}$ from the subsystem $k$ and the overall parallel system, respectively. Apparently, it finds that $\lambda_{k}$ increases if the subsystem $k$ has more importance in $\mathrm{DMU}_{0}$, and $\sum_{k=1}^{p} \lambda_{k}=1$.

Therefore, a following model can be used to evaluate the relative efficiency of an overall parallel system $\mathrm{DMU}_{0}$ : 


$$
\begin{array}{ll}
\max & e_{0}=\sum_{k=1}^{p}\left(\lambda_{k} \times e_{0}^{k}\right) \\
\text { s.t. } & \frac{\sum_{r=1}^{s} u_{r} Y_{r j}^{k}+\mu_{0}^{k}}{\sum_{i=1}^{m} w_{i} X_{i j}^{k}} \leq 1, \forall j, k \\
& w_{i}, u_{r} \geq 0, \forall r, i, \mu_{0}^{k}, \text { free }, \forall k
\end{array}
$$

In model (4), all the weights attached to the same inputs and outputs in both subsystems are replaced by $w_{i}$, $u_{r}$, as shown in (2). The sets of constraints ensure the efficiency of each subsystem to all DMUs is not more than one. The objective function of model (4) is the weighted objective function of each subsystem based upon model (1) as follows:

$$
e_{0}=\sum_{k=1}^{p}\left(\lambda_{k} \times e_{0}^{k}\right)
$$

Substitute (1) and (3) into (5), then the objective function of model (4) can be changed to be:

$$
\begin{aligned}
e_{0}= & \sum_{k=1}^{p}\left(\lambda_{k} \times e_{0}^{k}\right)=\frac{\sum_{i=1}^{m} w_{i} X_{i 0}^{1}}{\sum_{i=1}^{m} w_{i} X_{i 0}} \times \frac{\sum_{r=1}^{s} u_{r} Y_{r 0}^{1}+\mu_{0}^{1}}{\sum_{i=1}^{m} w_{i} X_{i 0}^{1}}+\cdots \\
& +\frac{\sum_{i=1}^{m} w_{i} X_{i 0}^{k}}{\sum_{i=1}^{m} w_{i} X_{i 0}} \times \frac{\sum_{r=1}^{s} u_{r} Y_{r 0}^{k}+\mu_{0}^{k}}{\sum_{i=1}^{m} w_{i} X_{i 0}^{k}}+\cdots+\frac{\sum_{i=1}^{m} w_{i} X_{i 0}^{p}}{\sum_{i=1}^{m} w_{i} X_{i 0}} \\
& \times \frac{\sum_{r=1}^{s} u_{r} Y_{r 0}^{p}+\mu_{0}^{p}}{\sum_{i=1}^{m} w_{i} X_{i 0}^{p} u_{r} Y_{r 0}+\sum_{k=1}^{p} \mu_{0}^{k}}
\end{aligned}
$$

Then the fractional program (4) can be written as follows:

$$
\begin{gathered}
\max e_{0}=\frac{\sum_{r=1}^{s} u_{r} Y_{r 0}+\sum_{k=1}^{p} \mu_{0}^{k}}{\sum_{i=1}^{m} w_{i} X_{i 0}} \\
\text { s.t. } \quad \frac{\sum_{r=1}^{s} u_{r} Y_{r j}^{k}+\mu_{0}^{k}}{\sum_{i=1}^{m} w_{i} X_{i j}^{k}} \leq 1, \forall j, k \\
w_{i}, u_{r} \geq 0, \forall r, i, \mu_{0}^{k}, \text { free, } \forall k .
\end{gathered}
$$

The above program (6) can be converted to the following model by applying the Charnes-Cooper (C-C) transformation:

$$
\begin{array}{ll}
\max & e_{0}=\sum_{r=1}^{s} \eta_{r} Y_{r 0}+\sum_{k=1}^{p} \omega_{0}^{k} \\
\text { s.t. } & \sum_{r=1}^{s} \eta_{r} Y_{r j}^{k}+\omega_{0}^{k}-\sum_{i=1}^{m} v_{i} X_{i j}^{k} \leq 0, \forall j, k \\
& \sum_{i=1}^{m} v_{i} X_{i 0}=1 \\
& v_{i}, \eta_{r} \geq 0, \forall r, i, \omega_{0}^{k}, \text { free }, \forall k .
\end{array}
$$

Denote the optimal objective function value of model (7) as $e_{0}$ which is the overall parallel efficiency score of $\mathrm{DMU}_{0}$. It is worth noted that if $\mu_{0}=0$, the model was also established, that is to say the model can be applied in the case of constant returns to scale.

Definition 1: The overall parallel system of $\mathrm{DMU}_{0}$ is said to be efficient if $e_{0}=1$.

Theorem 1: If the overall parallel system of $\mathrm{DMU}_{0}$ is efficient, then only if its each subsystem is efficient, namely $e_{0}^{k}=1, \forall k$.

Proof: First, we prove that $\lambda_{k}(k=1, \cdots, p)$ is a positive number which is bigger than 0 , but smaller than 1 .

First of all, take $\lambda$ as an example, If $\lambda_{1}=0$, then we obtain $\mu_{r}=0, \forall r$ according to definition (3). Under this condition, the denominator of (3) is 0 , which is meaningless, so $\lambda_{1} \neq 0$.

If $\lambda_{1}=1$, then we obtain $\lambda_{k}=0, \forall k \neq 1$, for $\sum_{k=1}^{p} \lambda_{k}=1$. In this case, the denominator of (3) for $\lambda_{k}=0, \forall k \neq 1$ is also 0 . Therefore, we have $\lambda_{k} \in(0,1), \quad(k=1, \cdots, p)$.

If $\mathrm{DMU}_{0}$ is efficient in overall parallel system $\left(e_{0}=1\right)$, then we obtain $\sum_{k=1}^{p} \lambda_{k} \times e_{k}=1$ based upon (5).

Similarly, suppose the subsystem 1 is non-efficient, in other words, $e_{0}^{1}<1$, Therefore, $e_{0}^{1} \times \lambda_{1}<\lambda_{1}$. However, according $e_{0}^{1} \times \lambda_{1}+\sum_{k=2}^{p} \lambda_{k} \times e_{k}=1$ and $e_{0}^{k} \in[0,1]$. if $e_{0}^{k}=1, \quad \forall k \neq 1, \sum_{k=2}^{p} \lambda_{k} \times e_{k}$ obtain the maximum value, then, $e_{0}^{1} \times \lambda_{1}+\sum_{k=2}^{p} \lambda_{k}=1$, due to $\sum_{k=1}^{p} \lambda_{\mathrm{k}}=1$ and $e_{0}^{1} \times \lambda_{1}<\lambda, \quad e_{0}^{1} \times \lambda_{1}+\sum_{k=2}^{p} \lambda_{k}=1$ can be set up only if $e_{0}^{1}=1$, if $\exists e_{0}^{k}<1, \forall k \neq 1$, if $e_{0}^{1} \times \lambda_{1}+\sum_{k=2}^{p} \lambda_{k}=1$ holds, requires $e_{0}^{1}>1$.

Apparently, it contradicts with the fact that $e_{0}^{k}(k=1, \cdots, p)$ is not less than 1 . Hence we prove that the subsystem 1 is efficient $\left(e_{0}^{1}=1\right)$.

The similar analysis can be applied to subsystem $k$, 
$(k=2, \cdots, p)$, so $e_{0}^{k}=1, \forall k$. Thus, the theorem has been completely proven.

\subsection{Models for Measuring Each Subsystem: An Efficiency Decomposition}

Based upon model (7), we can indirectly calculate the efficiency scores for each subsystem on the use of the optimal solution. However, the efficiency values of each subsystem may be non-unique, since the model (7) may have alternative optimal multipliers. Here we develop models of efficiency decomposition for each subsystem.

By substitution of each alternative optimal multiplier which is derived from the model (7), the maximum and minimum efficiencies values of each subsystem can be determined. Suppose the maximum and minimum values of each subsystem of $\mathrm{DMU}_{0}$ can be denoted as $\overline{e_{0}^{k}}$ and $\underline{e_{0}^{k}}(k=1, \cdots, p)$, respectively. Then they can be calculated though the models of following form:

$$
\begin{gathered}
\min / \max e_{0}^{k-d}=\frac{\sum_{r=1}^{s} u_{r} Y_{r 0}^{k}+\mu_{0}^{k}}{\sum_{i=1}^{m} w_{i} X_{i 0}^{k}} \forall k . \\
\text { s.t. } \frac{\sum_{r=1}^{s} u_{r} Y_{r 0}+\sum_{k=1}^{p} \mu_{0}^{k}}{\sum_{i=1}^{m} w_{i} X_{i 0}}=e_{0}^{*} \\
\sum_{r=1}^{s} u_{r} Y_{r j}^{k}+\mu_{0}^{k} \\
\sum_{i=1}^{m} w_{i} X_{i j}^{k} \leq 1, \forall j, k \\
w_{i}, u_{r} \geq 0, \forall r, i, \mu_{0}^{k}, \text { free, } \forall k .
\end{gathered}
$$

where $e_{0}^{*}$ is the optimal objective function value of model (7), and the first constraint ensures that the overall parallel system maintains its efficiency score invariant. Similarly, the non-linear program (8) can also be converted into a linear program via $\mathrm{C}-\mathrm{C}$ transformation as follows:

$$
\begin{array}{ll}
\min / \max e_{0}^{k-d}=\sum_{r=1}^{s} \eta_{r} Y_{r 0}^{k}+\omega_{0}^{k} \quad \forall k . \\
\text { s.t. } \quad \sum_{r=1}^{s} \eta_{r} Y_{r 0}+\sum_{k=1}^{p} \omega_{0}^{k}-e_{0}^{*} \sum_{i=1}^{m} v_{i} X_{i 0}=0 \\
& \sum_{r=1}^{s} \eta_{r} Y_{r j}^{k}+\omega_{0}^{k}-\sum_{i=1}^{m} v_{i} X_{i j}^{k} \leq 0, \forall j, k \\
& \sum_{i=1}^{m} v_{i} X_{i 0}^{k}=1 \\
& v_{i}, \eta_{r} \geq 0, \forall r, i, \omega_{0}^{k}, \text { free }, \forall k .
\end{array}
$$

To distinguish the objective function value in model (1), here we denote $e_{o}^{k-d}$ as objective function value. Denote the maximum and minimum objective function values as $\overline{e_{0}^{k}}$ and $e_{0}^{k}(k=1, \cdots, p)$ which can work out by the model (8). Therefore, the efficiency of each subsystem $k$ can be determined in an interval

$\left[\underline{e_{0}^{k}}, \overline{e_{0}^{k}}\right](k=1, \cdots, p)$. If $\overline{e_{0}^{k}}=\underline{e_{0}^{k}}, \exists k$ is satisfied, then we can conclude that subsystem $k$ has a unique efficiency value.

Theorem 2: If the overall parallel system of $\mathrm{DMU}_{0}$ is efficient, then $\underline{e_{0}^{k}}=\overline{e_{0}^{k}}=1, \forall k$

Proof: In the Theorem 1 which has proved an overall parallel system of $\mathrm{DMU}_{0}$ is efficient if and only if its each subsystem is efficient, namely $e_{0}^{k}=1, \forall k$.That is to say the subsystem has a unique solution equals to 1 , thus, the maximum and minimum efficiencies was only identified, namely $\underline{e_{0}^{k}}=\overline{e_{0}^{k}}=1, \forall k$. Then the Theorem 2 has proved.

\subsection{Numerical Example}

Here we use the example of Yang et al. (2000), the data is shown in Table 1. There are 4 DMUs and each has two subsystems which use single input to generate single output. Then we use the above models to calculate the efficiency values for each DMU as well as their each subsystem, the results are gave in Table 2, in addition, the third column of Table 2 is the results of the overall efficiency values for four DMUs when using YMK model. It can be seen that the efficiency values which are calculated by two kinds of models are different though the comparison, however, the common point is both the results reveal that only DMU 4 is efficient when evaluating the overall system, this proved that two models have discriminated the most effective DMU. Meanwhile,

Table 1. Data of an example.

\begin{tabular}{ccccc}
\hline & \multicolumn{2}{c}{ Input } & \multicolumn{2}{c}{ Output } \\
\hline DMU & Subsystem 1 & Subsystem 2 & Subsystem 1 & Subsystem 2 \\
1 & 1 & 3 & 1 & 2 \\
2 & 2 & 1 & 1 & 1 \\
3 & 1 & 3 & 2 & 2 \\
4 & 1 & 1 & 3 & 2 \\
\hline
\end{tabular}


Table 2. Efficiency values of the example.

\begin{tabular}{ccccc}
\hline DMU & $\begin{array}{c}\text { The overall efficiency value } \\
\text { (model 7) }\end{array}$ & $\begin{array}{c}\text { The overall efficiency value } \\
\text { (YMK model) }\end{array}$ & $\begin{array}{c}\text { The efficiency value of } \\
\text { subsystem 1 }\end{array}$ & $\begin{array}{c}\text { The efficiency value of } \\
\text { subsystem } 2\end{array}$ \\
\hline 1 & 0.5 & 0.33 & 1 & 0.3333 \\
2 & 0.6667 & 0.5 & 0.5 & 1 \\
3 & 0.5 & 0.667 & 1 & 0.3333 \\
4 & 1 & 1 & 1 & 1 \\
\hline
\end{tabular}

based upon models of efficiency decomposition, each subsystem was proved that they have unique solution. The result of DMU 4 illustrates the Theorem 1 as it shows that the efficiency value of each subsystem is 1 . In addition, for other three inefficient DMUs, only one of their subsystems is efficient, this brings about monolithic inefficient. Thus, taking internal structure of the DMU into consideration is necessary for efficiency evaluation of the overall system. Besides, take DMU 1 as an example, subsystem 2 is considered more important as the majority of the inputs are allocated to it, if we don not take the importance of subsystem 2 into consideration, the efficiency value of subsystem 2 is more than 0.3333 . As subsystem 2 is non-effective, the decision maker should transfer some inputs to subsystem 1 for improving the overall performance.

\section{Application to Technological Innovation Organization of Each Region in China}

China has an increasing development in the area of technology since the economic reform in 1978, and these technological activities have a great contribution to the economic growth and social development. However, each province in china demonstrated different efficiencies in technological innovation activities. Zhong et al. (2010) utilize (DEA) models to evaluate the relative efficiencies of 30 regions in China, they indicated that technological innovation development was unbalance in each region. In this paper, we believe that the major technological innovation department of each province includes three portions: R \& D (Research and Development) Institutions, Large \& Medium-sized Enterprises and Institutions of Higher Education. They were considered as a parallel structure as we described before.

\subsection{Selection of Inputs and Outputs and Data}

Many different inputs and outputs indices were selected to evaluate the technological innovation activity. However, it is well known that the discrimination power of DEA models will be much weakened if too many inputs or outputs indicators are used [17], we should chose the factors that can fully characterize the impact on the performance of technological innovation activity. Zhong [18] used R \& D expenditure and Full-time equivalent of R \& $\mathrm{D}$ personnel as inputs and Patent applications, the sales revenue of new products and the profit of primary business as outputs, nevertheless, according to Griliches [19], Ahuja and Katila [20], R \& D expenditure and R \& D personnel, Patent applications and the sales revenue of new products are the most important inputs and outputs of technological innovation activity, respectively. In this paper, the two kinds of core inputs, R \& D expenditure and $\mathrm{R} \& \mathrm{D}$ personnel, were represent by Intramural Expenditure on R \& D (: $1000 \mathrm{RMB} \$$ ) and Full-time equivalent of $\mathrm{R} \& \mathrm{D}$ (: man-year) personnel, respectively. The number of Patent granted (: item) is more appropriate to reflect the outputs of technological innovation activity, the sales revenue of new products (: $1000 \mathrm{RMB} \$$ ), an index that directly measures product innovation, refers to the sales revenue achieved from sales of new products in the reporting year. Thus, we have two inputs and two outputs.

The data we used are 30 regions of China in 2008 with an exception of Tibet, because of the missing statistical data. The data is obtained from the China Statistical Yearbook 2009 and China Statistical Yearbook on science and technology 2009. So we omited here.

\subsection{Improvement with the Model}

Noted that data of outputs, we could not acquire the precise outputs values to each organization in each province. Actually in China, the outputs of the technological innovation derive from the combination among these three organizations. However, in the daily management, each organization operated independently. The school commits itself to achieve theoretical innovation and breakthrough of key technologies, R \& D institutions transform the scientific research achievements into products, the enterprise yields and sales the products and then gains profit. This indicated that the output of the technological innovation is an outcome of three organizations. 
Thus, we give the whole outputs data.

To solve this problem, we assumed that the overall outputs $Y_{i j}(i=1,2)$ are divided into three portions to each organization.

$$
Y_{i j}^{1}=\alpha \times Y_{i j}, \quad Y_{i j}^{2}=\beta \times Y_{i j} \text { and } Y_{i j}^{3}=(1-\alpha-\beta) \times Y_{i j}
$$

Based upon model (7), by substituting (10) into (7), then the overall parallel system can be evaluated by following model:

$$
\begin{array}{ll}
\max & e_{0}=\sum_{r=1}^{s} \eta_{r} Y_{r 0}+\omega_{0}^{1}+\omega_{0}^{2}+\omega_{0}^{3} \\
\text { s.t. } & \sum_{r=1}^{s} \alpha \times \eta_{r} Y_{r j}+\omega_{0}^{1}-\sum_{i=1}^{m} v_{i} X_{i j}^{1} \leq 0, \forall j \\
& \sum_{r=1}^{s} \beta \times \eta_{r} Y_{r j}+\omega_{0}^{2}-\sum_{i=1}^{m} v_{i} X_{i j}^{2} \leq 0, \forall j \\
& \sum_{r=1}^{s}(1-\alpha-\beta) \times \eta_{r} Y_{r j}+\omega_{0}^{3}-\sum_{i=1}^{m} v_{i} X_{i j}^{3} \leq 0, \forall j \\
& \sum_{i=1}^{m} v_{i}\left(X_{i 0}^{1}+X_{i 0}^{2}+X_{i 0}^{3}\right)=1 \\
& 0 \leq \alpha, \beta,(1-\alpha-\beta) \leq 1 \\
& v_{i}, \eta_{r} \geq 0, \forall r, i, \omega_{0}^{k}, \text { free }, \forall k .
\end{array}
$$

The above model (11) is a non-linear programming, let $\alpha \times \eta_{r}=\pi_{r}, \beta \times \eta_{r}=\sigma_{r}$, then we can convert it to a linear programming as follows:

$$
\begin{aligned}
\max & e_{0}=\sum_{r=1}^{s} \eta_{r} Y_{r 0}+\omega_{0}^{1}+\omega_{0}^{2}+\omega_{0}^{3} \\
& \sum_{r=1}^{s} \pi_{r} Y_{r j}-\sum_{i=1}^{m} v_{i} X_{i j}^{1}+\omega_{0}^{1} \leq 0, \forall j \\
& \sum_{r=1}^{s} \sigma_{r} Y_{r j}-\sum_{i=1}^{m} v_{i} X_{i j}^{2}+\omega_{0}^{2} \leq 0, \forall j \\
& \sum_{r=1}^{s} \eta_{r} Y_{r j}-\sum_{r=1}^{s} \pi_{r} Y_{r j}-\sum_{r=1}^{s} \sigma_{r} Y_{r j}-\sum_{i=1}^{m} v_{i} X_{i j}^{3}+\omega_{0}^{3} \leq 0, \forall j \\
& \sum_{i=1}^{m} v_{i}\left(X_{i 0}^{1}+X_{i 0}^{2}+X_{i 0}^{3}\right)=1 \\
& \eta_{r} \geq \pi_{r} ; \eta_{r} \geq \sigma_{r}, \forall r \\
& v_{i}, \eta_{r}, \pi_{r}, \sigma_{r} \geq 0, \forall r, i, \omega_{0}^{1}, \omega_{0}^{2}, \omega_{0}^{3}, \text { free }
\end{aligned}
$$

By solving model (12), the efficiency value of the overall system can be obtained. Similarly, we applied the same method to the models for measuring the efficiency of each subsystem. The linear programming is showed in model (13).

$$
\max / \min e_{0}^{k}=\sum_{r=1}^{s} \eta_{r} Y_{r 0}^{k}+\omega_{0}^{k}, \forall k=1,2,3
$$

$$
\begin{aligned}
& \sum_{r=1}^{s} \pi_{r} Y_{r j}-\sum_{i=1}^{m} v_{i} X_{i j}^{1}+\omega_{0}^{1} \leq 0, \forall j \\
& \sum_{r=1}^{s} \sigma_{r} Y_{r j}-\sum_{i=1}^{m} v_{i} X_{i j}^{2}+\omega_{0}^{2} \leq 0, \forall j \\
& \sum_{r=1}^{s} \eta_{r} Y_{r j}-\sum_{r=1}^{s} \pi_{r} Y_{r j}-\sum_{r=1}^{s} \sigma_{r} Y_{r j}-\sum_{i=1}^{m} v_{i} X_{i j}^{3}+\omega_{0}^{3} \leq 0, \forall j \\
& \sum_{i=1}^{m} v_{i} X_{i 0}^{k}=1, k=1,2,3 \\
& \sum_{r=1}^{s} \eta_{r} Y_{r 0}-e_{0}^{*} \sum_{i=1}^{m} v_{i} X_{i 0}+\omega_{0}^{1}+\omega_{0}^{2}+\omega_{0}^{3}=0 \\
& u_{r} \geq \pi_{r} ; u_{r} \geq \sigma_{r}, \forall r \\
& w_{i}, u_{r}, \pi_{r}, \sigma_{r} \geq 0, \forall r, i, \omega_{0}^{k}, \text { free }, k=1,2,3
\end{aligned}
$$

\subsection{Results}

Based upon the data, we applied model (12) to evaluate the overall efficiencies of technological innovation in each region, the results are shown in the second column of the Table 3. The efficiencies of BCC model which did not take the internal structure into consideration was shown in the first column for compare. By analyzing the two computational results, we can find that the BCC efficiency values are larger than model (12), this is due to the model (12) has strong restriction which each technological innovation organization are compared, rather than compared only with region. While using BCC model for measuring, there are seven efficient regions which are Tianjin, Jilin, Shanghai, Jiangsu, Zhejiang, Guangdong and Hainan. However, five of them are no longer efficient in our evaluating method, only two regions are keep efficient, they are Zhejiang and Guangdong, for these five regions, Jilin has the greatest impact, as we can see its efficiency value is only 0.6736 when we take each technological innovation organization into account. In addition, for other inefficient regions, Shangxi has the smallest efficiency value, this is same as the conclusion which can be given by using BCC model, besides, the efficiency value of Qinghai has the maximum reduction, as the value decrease 0.3067 , and the most possible reason may be the irrational resource allocation of each organization.

Based upon model (13), the efficiency decomposition results have shown in the Table 4 . The last three columns are the maximum and minimum efficiency values of each agency, respectively. From the table, we noted the range that the efficiency values change is extremely small, and the maximum deviations are only 0.0086 (Liaoning) 0.0109 (Sichuan), 0.0493 (Hunan) in each organization, respectively. Thus we can conclude that the majority of the regions have a unique efficiency value 
Table 3. Efficiency measures of technological innovation.

\begin{tabular}{|c|c|c|}
\hline Region & BCC model & Model (12) \\
\hline Anhui & 0.4108 & 0.2853 \\
\hline Beijing & 0.3434 & 0.2845 \\
\hline Chongqing & 0.9418 & 0.6544 \\
\hline Fujian & 0.7790 & 0.5987 \\
\hline Gansu & 0.3087 & 0.2004 \\
\hline Guangdong & 1 & 1 \\
\hline Guangxi & 0.6820 & 0.4849 \\
\hline Guizhou & 0.4928 & 0.3924 \\
\hline Hainan & 1 & 0.7500 \\
\hline Hebei & 0.4454 & 0.3244 \\
\hline Heilongjiang & 0.2603 & 0.2353 \\
\hline Henan & 0.4739 & 0.3855 \\
\hline Hubei & 0.4970 & 0.3593 \\
\hline Hunan & 0.5254 & 0.3757 \\
\hline Inner Mongolia & 0.3864 & 0.2591 \\
\hline Jiangsu & 1 & 0.9201 \\
\hline Jiangxi & 0.4271 & 0.2744 \\
\hline Jilin & 1 & 0.6736 \\
\hline Liaoning & 0.4588 & 0.3423 \\
\hline Ningxia & 0.6147 & 0.3874 \\
\hline Qinghai & 0.8433 & 0.5366 \\
\hline Shaanxi & 0.1668 & 0.1436 \\
\hline Shandong & 0.8504 & 0.7346 \\
\hline Shanghai & 1 & 0.6782 \\
\hline Shanxi & 0.3768 & 0.2630 \\
\hline Sichuan & 0.4429 & 0.3906 \\
\hline Tianjin & 1 & 0.7706 \\
\hline Xinjiang & 0.5157 & 0.4037 \\
\hline Yunnan & 0.4079 & 0.3108 \\
\hline Zhejiang & 1 & 1 \\
\hline
\end{tabular}

when the minimal biases are neglected. For R \& D Institutions as an example, there are regions such as Shanxi, Zhejiang, Ningxia, Xinjiang and so on. In addition, the results of Table 4 show some regions have a good performance in one of the organizations but not the overall technological innovation organizations. These regions include Jilin, Shanghai, Zhejiang, Hainan and Ningxia, as it can be seen that efficiency scores of one of their subsystem can achieve to 1 , among these regions, only Ningxia is efficient in two subsystems, that is to say, if it want be efficient in overall organizations, only improved the activity of R \& D Institutions can achieve this goal. Apart form these, it is worth noted the two best performance region: Jiangsu and Guangdong. The efficiency value of each organization of them is equal to 1 , as it can be proved in theorem 1 and 2. According to the data, most of the resources are invested in Large \& Mediumsized Enterprises in region technological innovation activity, in other words, all these regions deemed the Large \& Medium-sized Enterprises more important, this phenomenon explained the fact that China devote itself to develop the economy, however, the technological innovation activity of Large \& Medium-sized Enterprises in most of regions are non-effective, each region should make rational resource allocation.

\section{Conclusions}

In this paper, we considered DMUs were composed of same several subsystems, then a parallel system DEA 
Table 4. Results for efficiency decomposition.

\begin{tabular}{|c|c|c|c|c|c|c|}
\hline \multirow{2}{*}{ Region } & \multicolumn{2}{|c|}{ R \& D Institutions } & \multicolumn{2}{|c|}{ Large \& Medium-sized Enterprises } & \multicolumn{2}{|c|}{ Institutions of Higher Education } \\
\hline & $\underline{e_{0}^{1}}$ & $\overline{e_{0}^{1}}$ & $\underline{e_{0}^{2}}$ & $\overline{e_{0}^{2}}$ & $\underline{e_{0}^{3}}$ & $\overline{e_{0}^{3}}$ \\
\hline Anhui & 0.0947 & 0.0955 & 0.3422 & 0.3432 & 0.2736 & 0.2769 \\
\hline Beijing & 0.0312 & 0.0316 & 0.9877 & 0.9882 & 0.2022 & 0.2032 \\
\hline Chongqing & 0.8415 & 0.8418 & 0.6835 & 0.6835 & 0.4518 & 0.4520 \\
\hline Fujian & 0.6464 & 0.6469 & 0.5777 & 0.5780 & 0.8180 & 0.8216 \\
\hline Gansu & 0.0616 & 0.0619 & 0.2692 & 0.2710 & 0.2265 & 0.2349 \\
\hline Guangdong & 1 & 1 & 1 & 1 & 1 & 1 \\
\hline Guangxi & 0.3107 & 0.3107 & 0.5456 & 0.5456 & 0.3444 & 0.3444 \\
\hline Guizhou & 0.2338 & 0.2338 & 0.4065 & 0.4132 & 0.4608 & 0.4809 \\
\hline Hainan & 0.4601 & 0.4601 & 1 & 1 & 1 & 1 \\
\hline Hebei & 0.1591 & 0.1668 & 0.3540 & 0.3541 & 0.3454 & 0.3610 \\
\hline Heilongiiang & 0.0723 & 0.0760 & 0.3361 & 0.3363 & 0.0961 & 0.1002 \\
\hline Henan & 0.1446 & 0.1449 & 0.4110 & 0.4111 & 0.6623 & 0.6633 \\
\hline Hubei & 0.0822 & 0.0826 & 0.5110 & 0.5122 & 0.2454 & 0.2489 \\
\hline Hunan & 0.215 & 0.2159 & 0.4336 & 0.4383 & 0.2108 & 0.2601 \\
\hline Inner Mongolia & 0.2276 & 0.2282 & 0.2343 & 0.2344 & 0.8971 & 0.9093 \\
\hline Jiangsu & 0.3706 & 0.3778 & 1 & 1 & 0.7985 & 0.8056 \\
\hline Jiangxi & 0.1870 & 0.1939 & 0.2929 & 0.2929 & 0.2734 & 0.2780 \\
\hline Jilin & 0.2104 & 0.2105 & 1 & 1 & 0.4229 & 0.4240 \\
\hline Liaoning & 0.1433 & 0.1519 & 0.4220 & 0.4221 & 0.2526 & 0.2636 \\
\hline Ningxia & 1 & 1 & 0.2727 & 0.2727 & 0.9249 & 0.9251 \\
\hline Qinghai & 0.5871 & 0.5871 & 0.5319 & 0.5319 & 0.5037 & 0.5037 \\
\hline Shaanxi & 0.0239 & 0.0245 & 0.2637 & 0.2685 & 0.1472 & 0.1488 \\
\hline Shandong & 0.6151 & 0.6157 & 0.7253 & 0.7286 & 0.8899 & 0.9987 \\
\hline Shanghai & 0.1058 & 0.1065 & 1 & 1 & 0.4217 & 0.4252 \\
\hline Shanxi & 0.1661 & 0.1663 & 0.2669 & 0.2669 & 0.3681 & 0.3683 \\
\hline Sichuan & 0.0408 & 0.0432 & 0.8158 & 0.8267 & 0.2086 & 0.2187 \\
\hline Tianjin & 0.4643 & 0.4651 & 0.8701 & 0.8706 & 0.6856 & 0.6877 \\
\hline Xinjiang & 0.1805 & 0.1805 & 0.4277 & 0.4277 & 0.6649 & 0.6649 \\
\hline Yunnan & 0.0462 & 0.0465 & 0.6427 & 0.6435 & 0.2454 & 0.2462 \\
\hline Zhejiang & 1 & 1 & 1 & 1 & 1 & 1 \\
\hline
\end{tabular}

model was present, to distinguish with other DEA literatures, we endow each subsystem with a weight which was considered as different importance of each subsystem. After the efficiency of overall parallel system has been obtained, we also develop models of efficiency decomposition to determine the interval of the efficiency value of each subsystem. Finally, we applied the improvement models to evaluate the principal technological innovation organization of each region in China, the results indicated that only Zhejiang and Guangdong are efficient in overall technological innovation system, besides, the results also point out the direction for efficiency improvement.

\section{Acknowledgements}

This research is supported by the National Natural Science Foundation of China under Grants (No. 70901070, 70821001) and Postdoctoral Science Foundation of China under Grants (No. 200902297, 20080440714).

\section{References}

[1] Y. J. Li, F. Yang, L. Liang and Z. S. Hua, "Allocating the Fixed Cost as a Complement of Other Cost Inputs: A DEA Approach," European Journal of Operational Research, Vol. 197, No. 1, 2009, pp. 389-401. doi:10.1016/j.ejor.2008.06.017 
[2] A. Charnes, W. W. Cooper and E. Rhodes, "Measuring the Efficiency of Decision Making Units," European Journal of Operational Research, Vol. 2, No. 6, 1978, pp. 429-444. doi:10.1016/0377-2217(78)90138-8

[3] R. D. Banker, A. Charnes and W. W. Cooper, "Some Models for Estimating Technical and Scale Inefficiencies in Data Envelopment Analysis," Management Science, Vol. 30, No. 9, 1984, pp. 1078-1092. doi:10.1287/mnsc.30.9.1078

[4] R. Färe and S. Grosskopf, "A Nonparametric Cost Approach to Scale Efficiency," Journal of Economics, Vol. 87, No. 4, 1985, pp. 594-604.

[5] L. M. Seiford and R. M. Thrall, "Resent Development in DEA-The Mathematical Programming Approach to Frontier Analysis," Journal of Economics, Vol. 46, No. 1-2, 1990, pp. 7-38.

[6] C. Kao and S. N. Hwang, "Efficiency Decomposition in Two-Stage Data Envelopment Analysis: An Application to Non-Life Insurance Companies in Taiwan," European Journal of Operational Research, Vol. 185, No. 1, 2008, pp. 418-429. doi:10.1016/j.ejor.2006.11.041

[7] L. Liang, W. D. Cook and J. Zhu, "DEA Models for Two-Stage Processes: Game Approach and Efficiency Decomposition," Naval Research Logistics, Vol. 55, No. 7, 2008, pp. 643-653. doi:10.1002/nav.20308

[8] Y. Chen, J. Du, H. D. Sherman and J. Zhu, "DEA Model with Shared Resources and Efficiency Decomposition," European Journal of Operational Research, Vol. 207, No. 1, 2010, pp. 339-349.

[9] Y. Zha and L. Liang, "Two-Stage Cooperation Model with Input Freely Distributed among the Stages," European Journal of Operational Research, Vol. 205, No. 2, 2010, pp. 332-338. doi:10.1016/j.ejor.2010.01.010

[10] R. Färe and S. Grosskopf, "Network DEA," Socio-Economic Planning Science, Vol. 34, No. 1, 2000, pp. 35-49. doi:10.1016/S0038-0121(99)00012-9

[11] H. F. Lewis and T. R. Sexton, "Network DEA: Efficiency Analysis of Organizations with Complex Internal Structure," Computers and Operations Research, Vol. 31, No. 9, 2004, pp. 1365-1410.
doi:10.1016/S0305-0548(03)00095-9

[12] K. Tone and M. Tsutsui, "Network DEA: A Slacks-Based Measure Approach," European Journal of Operational Research, Vol. 197, No. 1, 2009, pp. 243-252. doi:10.1016/j.ejor.2008.05.027

[13] R. Färe and D. Primont, "Efficiency Measures for Multiplant Firms," Operations Research Letters, Vol. 3, No. 5, 1984, pp. 257-260. doi:10.1016/0167-6377(84)90057-9

[14] Y. S. Yang, B. J. Ma and M. Koike, "Efficiency-Measuring DEA Model for Production System with k Independent Subsystems," Journal of the Operations Research Society of Japan, Vol. 43, No. 3, 2000, pp. 343353.

[15] L. Castelli, R. Pesenti and W. Ukovich, "DEA-Like Models for the Efficiency Evaluation of Hierarchically Structured Units," European Journal of Operational Research, Vol. 154, No. 2, 2004, pp. 465-476. doi:10.1016/S0377-2217(03)00182-6

[16] C. Kao, "Efficiency Measurement for Parallel Production Systems," European Journal of Operational Research, Vol. 196, No. 3, 2009, pp. 1107-1112. doi:10.1016/j.ejor.2008.04.020

[17] W. Meng, D. Q. Zhang, L. Qi and W. B. Liu, "Two-Level DEA Approached in Research Evaluation," Omega, Vol. 36, No. 6, 2008, pp. 950-957. doi:10.1016/j.omega.2007.12.005

[18] W. Zhong, W. Yuan, S. X. Li and Z. M. Huang, "The Performance Evaluation of Regional R\&D Investments in China: An Application of DEA Based on the First Official China Economic Census Data," Omega, Vol. 39, No. 4, 2011, pp. 447-455. doi:10.1016/j.omega.2010.09.004

[19] Z. Griliches, "Patents Statistics as Economic Indicators: A Survey," Journal of Economic Literature, Vol. 28, No. 4, 1990, pp. 1661-1707.

[20] G. Ahuja and R. Karila, "Technological Acquisition and the Innovation Performance of Acquiring Firms: A Longitudinal Study," Strategic Management Journal, Vol. 22, No. 3, 2001, pp. 197-220. doi:10.1002/smj.157 